\title{
LA INVESTIGACIÓN EN LA UACh: balance preliminar y tendencias inmediatas
}

\author{
Liberio Victorino Ramírez \\ Universidad Autónoma de Chapingo
}

\section{INTRODUCCIÓN}

Mucho se dice respecto a la investigación en las universidades mexicanas. Destacan dos visiones: aquella que desconoce realmente lo que se investiga en las universidades y por tanto, afirman que no hay investigación en estas casas de estudios; otra corriente de opinión agrupa a los docentes y funcionarios que dignifican la función de investigación y en consecuencia sostienen que todo está bien que no hay nada por cambiar.

Lo que a continuación se presenta es precisamente un balance preliminar sobre el quehacer investigativo en la Universidad Autónoma Chapingo (UACh), particularmente a partir del análisis de fuentes directas referentes a proyectos, líneas y organización en general de la UACh. Dicho trabajo, presentado en una apretada síntesis, pretende dar una visión fundamentada, sin importar tanto influir en el balance sino más bien respetar el diagnóstico y las propias tendencias.

Desde luego existe la firme intención de profundizar más en el análisis, para que a mediano plazo se puedan plantear las mejores opciones en este campo, sobre todo que, sin salirse del contexto de globalización puedan atenderse prioritariamente el estudio de los grandes problemas nacionales,

El desarrollo de este trabajo, incluye los siguientes rubros: 1. Los profesores investigadores y el tipo de investigación que realizan; 2. Repercusión de los proyectos en el ámbito académico, agronómico y científico; 3, Formación de investigadores y su impacto en un sector de la comunidad científica; 4 . Tendencias de la investigación en la UACh; y 5.La investigación de vanguardia tecnológica. Para finalizar con las conclusiones. 


\section{LOS PROFESORES INVESTIGADORES Y EL TIPO DE INVESTIGACIÓN QUE REALIZAN}

En toda la Universidad ha variado el porcentaje de profesores que hacen investigación. Para 1990 sólo el 35\% de la planta del personal académico participa en proyectos de investigación. Aproximadamente 434 investigaciones, tomando como base que por lo menos un investigador participa en cada proyecto, se puede decir que son por lo menos 440 profesores investigadores como partícipes en proyectos de investigación, ya sea como responsable o bien como colaboradores de los proyectos (véase el Cuadro 1).

En términos relativos se puede pensar que para 1993 el porcentaje crece a un $40 \%$ aproximadamente, índice superior al año anterior. Existe la posibilidad que para 1995 este porcentaje se incremente; sin embargo, ello estará condicionado por apoyos frescos que otorguen las autoridades centrales de la UACh.

Aunque es difícil precisar la orientación de la investigación, se puede decir que el tipo de proyecto está asociado al tipo de investigación que, vienen desarrollando los profesores investigadores. Sin embargo, haciendo una rápida revisión, se puede señalar lo siguiente: Por sus características, enfoques y por la elaboración de conceptos, así como el planteamiento de problemas objetos de investigación, se pueden clasificar en: básica, aplicada y tecnológica. Atendiendo a la gran concentración de los proyectos en algunos DEIS como son: Fitotecnia, fundamentalmente, se puede decir que hay un predominio del tipo de investigación de rasgos tecnológicos y en menor cantidad en los otros dos tipos de investigación. Atendiendo la clasificación por área que tiene la SIS UACh, éstas se organizan en: producción forestal, ciencias sociales, ciencias naturales, producción agrícola, producción agroindustrial, producción pecuaria y ciencias exactas. Más claramente, la investigación de la UACh se ubica por la clasificación de las ciencias en: exactas, naturales y sociales, y por el tipo de producción en: agrícola, agro industrial, pecuaria y forestal. 


\section{CUADRO 1}

NUMERO DE INVESTIGACIONES POR INSTANCIAS 1990.

\begin{tabular}{lc}
\hline INSTANCIAS & No.INVEST. \\
\hline DICIFO & 22 \\
\hline ECONOMÍA AGRÍCOLA & 52 \\
\hline FITOTECNIA & 68 \\
\hline INDUSTRIAS AGRÍCOLAS & 20 \\
\hline IRRIGACIÓN & 39 \\
\hline MAQUINARIA AGRÍCOLA & 18 \\
\hline PARASITOLOGÍA AGRÍCOLA & 33 \\
\hline PREPARATORIA AGRÍCOLA & 12 \\
\hline SOCIOLOGÍA RURAL & 06 \\
\hline SUELOS & 20 \\
\hline ZONAS ÁRIDAS & 46 \\
\hline ZOOTECNIA & 21 \\
\hline CENTROS REGIONALES & 29 \\
\hline DETCU & 5 \\
\hline DIFUSIÓN CULTURAL & 34 \\
\hline PIIAI & $* 09$ \\
\hline SUBDIRECCION DE INVESTIGACIÓN & 434
\end{tabular}

${ }^{*}$ Incluye los Departamentos de Diagnóstico Externo, Coordinación de Servicio y Campo Experimental.

FUENTE: UPOM, UACh, 1990. 


\section{CUADRO 2}

PROYECTOS POR DEIS. 1993.

\begin{tabular}{lc}
\hline DEIS & Cantidad \\
\hline CENTROS REGIONALES & 17 \\
\hline CIESTAAM & 4 \\
\hline FITOTECNIA & 27 \\
\hline INGENIERÍA AGRÍCOLA & 4 \\
\hline PARASITOLOGÍA & 13 \\
\hline SIS UACh & 1 \\
\hline URUZA & 2 \\
\hline CIENCIAS FORESTALES & 7 \\
\hline ECONOMÍA AGRÍCOLA & 11 \\
\hline INGENIERÍA INDUSTRIAL & 3 \\
\hline IRRIGACIÓN & 8 \\
\hline PREPARATORIA AGRÍCOLA & 13 \\
\hline SOCIOLOGÍA RURAL & 23 \\
\hline ZOOTECNIA & 11 \\
\hline TOTAL & 144 \\
\hline
\end{tabular}

FUENTE: Elaboración propia con datos proporcionados por la SIS UACh.

Dentro del tratamiento para la investigación, sobre todo para la prioridad en la distribución del presupuesto, juegan un papel fundamental las tesis de licenciaturas de los estudiantes de los distintos DEIS, ello se refleja en la cantidad de tesis apoyadas económicamente por la UACh. 
CUADRO 3

PROYECTOS FINANCIADOS EN SU MODALIDAD DE TESIS DE LICENCIATURA 1993

\begin{tabular}{lc}
\hline DEIS & CANTIDAD \\
\hline CIENCIAS FORESTALES & 9 \\
\hline FITOTECNIA & 62 \\
\hline INGENIERÍA AGROINDUSTRIAL & 26 \\
\hline PARASITOLOGÍA & 20 \\
\hline SUELOS & 39 \\
\hline CENTROS REGIONALES & 6 \\
\hline ECONOMÍA AGRÍCOLA & 44 \\
\hline INGENIERÍA MECÁNICA AGRÍCOLA & 30 \\
\hline IRRIGACIÓN & 19 \\
\hline SOCIOLOGÍA RURAL & 1 \\
\hline PREPARATORIA AGRÍCOLA & 3 \\
\hline TOTAL & 259 \\
\hline
\end{tabular}

FUENTE: Elaboración propia con datos proporcionados por la SIS UACh. 


\section{CUADRO 4}

PROYECTOS FINANCIADOS POR LA UACh EN SU MODALIDAD DE PROYECTOS DE INVESTIGACIÓN DE PROFESORES. 1993

\begin{tabular}{lc}
\hline DEIS & CANTIDAD \\
\hline AGROECOLOGIA & 2 \\
\hline CIESTAAM & 2 \\
\hline ECONOMÍA AGRÍCOLA & 17 \\
\hline MAQUINARIA AGRÍCOLA & 1 \\
\hline P ARASITOLOGÍA & 11 \\
\hline SOCIOLOGÍA RURAL & 13 \\
\hline SIS UACh & 1 \\
\hline ZOOTECNIA & 16 \\
\hline CENTROS REGIONALES & 34 \\
\hline CIENCIAS FORESTALES & 17 \\
\hline INGENIERÍA AGROINDUSTRIAL & 8 \\
\hline IRRIGACIÓN & 4 \\
\hline PREPARATORIA AGRÍCOLA & 26 \\
\hline SUELOS & 8 \\
\hline URUZA & 13 \\
\hline FITOTECNIA & 2 \\
\hline TOTAL & 175 \\
\hline
\end{tabular}

FUENTE: Elaboración propia con datos proporcionados por la SIS UACh.

Si se hiciera una comparación del presupuesto universitario dedicado a la investigación, éste a partir de 1989, se ha venido incrementando paulatinamente (según se muestra en el Cuadro 5). Pese a sus limitaciones presupuestales que se dan al interior de la UACh, es importante reconocer que el presupuesto universitario dedicado a la investigación está por arriba de la media nacional. Mientras que la media nacional de las inversiones públicas fluctúa entre un 4 y 6\%, en la UACh, durante los últimos años, según la SIS- UACh, alcanza aproximadamente un $9 \%$ del presupuesto total. 
CUADRO 5

FINANCIAMIENTO OTORGADO A LA INVESTIGACIÓN DE LAS DIFERENTES INSTANCIAS O PROGRAMAS. AÑOS $1989 \mathrm{Y}$ 1990

\begin{tabular}{lrr}
\hline INSTANCIA O PROGRAMA & 1989 & 1990 \\
\hline DICIFO & 47100000 & 41183000 \\
\hline ECONOMÍA AGRÍCOLA & 88295800 & 124162800 \\
\hline FITOTECNIA & 314367210 & 150534460 \\
\hline INDUSTRIAS AGRÍCOLAS & 6157040 & 35603000 \\
\hline IRRIGACIÓN & 170400000 & 18500000 \\
\hline MAQUINARIA AGRÍCOLA & 25664800 & 92328600 \\
\hline PARASITOLOGÍA & 114261590 & 91060850 \\
\hline PREPARATORIA AGRÍCOLA & 75850500 & 62352083 \\
\hline SOCIOLOGÍA RURAL & 48322000 & 52236860 \\
\hline SUELOS & 114705200 & 30715650 \\
\hline ZONAS ARIDAS & 119601000 & 141546000 \\
\hline ZOOTECNIA & 228146500 & 463669630 \\
\hline DIAGNOSTICO EXTERNO & 21275000 & \\
\hline DETCU & 14819500 & 48000000 \\
\hline CENTROS REGIONALES & 162824000 & 93433334 \\
\hline SUBDIRECCION INV. & 12250781 & 98576100 \\
\hline PROG.INV.AGR.FOREST. & 15000000 & \\
\hline PIIAI & 156423290 & 171444820 \\
\hline T O TAL & 815964100 & 1822340100 \\
\hline
\end{tabular}

FUENTE: UPOM, UACh, 1990.

*Información no disponible. 


\section{REPERCUSIÓN DE LOS PROYECTOS EN EL ÁMBITO ACA- DÉMICO, AGRONÓMICO Y CIENTÍFICO}

En lo general, se puede decir que sí existe una repercusión académica endógena en la UACh. La prueba más elocuente es la significativa cantidad de tesistas de la UACh que están vinculados a profesores investigadores de los DEIS (véase el Cuadro 3). Otro aspecto que se refleja en la repercusión académica de los investigadores de la UACh, son el número de citas de los trabajos publicados en cuanto a la incorporación de sus propuestas teórico prácticas en los nuevos trabajos de investigación, esencialmente los tesistas de licenciatura y postgrado. En el área de Ciencias Socia- les, un elocuente ejemplo lo representa los trabajos, sobre regionalización en la agronomía del profesor Duch Gary de Centros Regionales, quien es uno de los autores más citados por profesores y alumnos, e incluso al interior de la Preparatoria Agrícola de la UACh.

En el ámbito agronómico se puede decir que toda- vía es más limitado que en el anterior punto. De lo que se conoce como impacto en el ámbito agronómico, destacan las recientes experiencias de los Programas Nacionales de Investigación sobre todo por las características de los proyectos colectivos e interinstitucionales. La trascendencia de los programas, se da como la expresión de varios años de trabajo antes de agruparse como alternativa de Programas Nacionales.

Por señalar algunos ejemplos, se pueden citar el Programa sobre el maíz: especialmente sus estudios sobre variedades de maíz. Asimismo se puede señalar el del Frijol; el de producción animal, etc. En el ámbito de las Ciencias Sociales destacan: el Programa Nacional de Investigación en Educación Agrícola, con una serie de proyectos interinstitucionales con otras Universidades de la región Zona Centro y de varias regiones del país y con escuelas y facultades del sector agronómico.

El impacto en este rubro se refiere al ámbito agro- nómico, entendiéndose a la agronomía como una disciplina o área de conocimiento que incluye a las ciencias exactas, naturales y sociales. Por lo que respecta a la repercusión en el terreno propiamente científico, se especifica en este caso en dos aspectos fundamentales muy ligado al circuito de relaciones científicas de la comunidad científica del área agronómica. Estos aspectos, son: la difusión del conocimiento científico y la formación de investigadores, así como su relación con las comunidades científicas institucionalizada en los órganos que deciden las políticas científicas nacionales; especialmente, el Sistema Nacional de Investigadores (SNI), y el CONACYT. 
Respecto al aspecto de la difusión del conocimiento científico, pese a los obstáculos en la demora de las publicaciones, las cuales duran hasta dos o tres años en salir a luz pública, la UACh ha mantenido $1 \mathrm{~m}$ nivel satisfactorio en las publicaciones especializadas según las áreas de conocimiento para la investigación. Aquí creo que el asunto de las publicaciones se ha mejorado en los últimos años, ya que si comparamos la producción a fines de los años ochentas con la que actualmente se tiene hay visos de cambios importantes. Desde hace más de 5 años que se institucionalizó la Feria del Libro de la UACh y con el apoyo de los Comités de publicaciones, integrado por profesores e investigadores de la UACh, se ha dinamizado un poco más la difusión del conocimiento científico.

\section{FORMACIÓN DE INVESTIGADORES Y SU IMPACTO EN LAS COMUNIDADES CIENTÍFICAS}

En lo referente a la formación de investigadores y a la relación de nuestra institución con el SNI y el CONACYT, hay coincidencia de varios autores en que la investigación y los procesos de formación de investigadores son procesos jóvenes en nuestro país, además de poco estudiado y de poco apoyo financiero y social de parte del Estado (De la Fuente, 1990; Ibarrola, 1990, Victorino, 1991).

Dentro del campo agronómico, especialmente en la UACh hay indicios de un incipiente desarrollo en el proceso de formación de investigadores. Primeramente es significativo el número de investigadores (léase los que hacen investigación) que desde 1990, alcanzan un importante porcentaje de la planta académica, sector que para 1993 y 1994 casi se incrementa, como se mostró al inicio de este ensayo. En segundo, y de manera intermitente destaca el nivel académico de cada uno de los investigadores, que también a partir de 1990 (véase el Cuadro 6), se ha venido incrementando, el cual, por ejemplo de 29 doctores que había en 1990 pasa a poco más de 70 entre 1993 y 1994. 
Convergencia Revista de Ciencias Sociales, núm. 8/9, 1995, Universidad Autónoma del Estado de México

\section{CUADRO 6}

NIVEL ACADÉMICO DE LOS INVESTIGADORES POR INSTANCIA. 1990

\begin{tabular}{|c|c|c|c|c|c|c|c|}
\hline INSTANCIA & P.LIC & LIC. & P.M.C. & M.C. & P.DOC & DOC. & TOTAL \\
\hline CENTROS REGIONALES & 2 & 23 & 1 & 7 & 3 & 0 & 36 \\
\hline DETCU & & 4 & & 2 & & & 6 \\
\hline DIAGNOSTICO EXTERNO & & 5 & & 2 & & & 7 \\
\hline DIFUSIÓN CULTURAL & & 2 & & & & & 2 \\
\hline DIV.CIENCIAS FORESTALES & 1 & 6 & 1 & 9 & & 6 & 23 \\
\hline ECONOMÍA AGRÍCOLA & & 6 & & 21 & 2 & 5 & 34 \\
\hline FITOTECNIA & & 17 & & 17 & & 5 & 29 \\
\hline INDUSTRIAS AGRÍCOLAS & & 10 & & 5 & & 2 & 17 \\
\hline IRRIGACIÓN & & 5 & & 7 & & 3 & 15 \\
\hline MAQUINARIA AGRÍCOLA & & 6 & 1 & 3 & & & 10 \\
\hline PIIAI & & 1 & & & & & 1 \\
\hline MAQUINARIA AGRÍCOLA & & & & 1 & & & 1 \\
\hline PARASITOLOGÍA AGRÍCOLA & & 6 & 1 & 14 & & 3 & 24 \\
\hline PREPARATORIA AGRÍCOLA & 1 & 13 & 4 & 11 & 2 & 1 & 32 \\
\hline SUBDIR. DE INVESTIGACIÓN & & 3 & 2 & 1 & & & 6 \\
\hline SUELOS & & 7 & 2 & 9 & 1 & & 19 \\
\hline ZONAS ARIDAS & & 9 & & 4 & 1 & & 14 \\
\hline TOTALES & 4 & 123 & 12 & 111 & 5 & & 29 \\
\hline
\end{tabular}

FUENTE: Elaboración propia con el apoyo de la Subdirección de Investigación de la UACh. 
En relación a los reconocimientos nacionales tanto premiados por el CONACYT como por la ocupación de una distinción nacional en el SNI, a pesar de sus vaivenes se ha logrado un desarrollo satisfactorio. De 25 profesores que lograron su ingreso en 1987 para 1993 había más de 100 investigadores en el SNI, principalmente en sus categorías de candidato a investigador nacional o bien como investigador nacional. Desde luego, no está por demás señalar que la permanencia en el SNI es fluctuante porque hay ingreso, reingreso, ascensos y bajas.

En la relación con el CONACYT, específicamente vías reconocimientos de premios nacionales y apoyo a Proyectos de Investigación y Organización de eventos nacionales e internacionales, ha sido poco significativo. Es decir si nuestro personal ha logrado premios nacionales y apoyos a proyectos de investigación y eventos, pero estos son poco atractivos en cantidad y en oportunidad. Por otro lado, casi no contamos con financiamiento a proyectos de investigación directa- mente del CONACYT, excepto uno en Ciencias Forestales para 1993. Este hecho muestra una gran desventaja ante otras instituciones como el Colegio de Postgrado, el cual un gran porcentaje de sus proyectos son apoyados por el CONACYT, o bien, la propia Universidad de San Luis Potosí, que también en el área agronómica, se les apoyó con casi el 80\% de sus proyectos que solicitaron financiamiento al CO-NACYT, en 1993.

Para especificar más sobre las repercusiones en el ámbito científico es ilustrativo el Cuadro 7 que muestra, especialmente el postgrado de la UACh y su impacto en el ámbito del reconocimiento científico en la formación de recursos humanos para la ciencia y la tecnología. También se aprecia esa fluctuación de los postgrados ante el Padrón de Excelencia, pues como se observa tanto en 1992 como en 1994, sólo el 50\% de los programas está incorporado en ese Padrón del CONACYT. 


\section{CUADRO 7}

POSTGRADO DE LA UACh ANTE EL PADRÓN DE EXCELENCIA DEL CONACYT. PERTENECE AL PADRÓN DE EXCELENCIA

\begin{tabular}{lccc}
\hline PROGRAMA & 1992 & 1993 & 1994 \\
\hline CIENCIAS FORESTALES & SI & SI & SI \\
\hline DESARROLLO RURAL REGIONAL & SI & SI & SI \\
\hline DOCTORADO EN ECONOMÍA AGRÍCOLA & NO & NO & NO \\
\hline ECONOMÍA DEL DESARROLLO RURAL & NO & SI & SI \\
\hline HORTICULTURA & NO & NO & \\
\hline PRODUCCIÓN ANIMAL & SI & NO & NO \\
\hline PROTECCIÓN VEGETAL & SI & NO & \\
\hline SOCIOLOGÍA RURAL & NO & NO & SI \\
\hline
\end{tabular}

FUENTE: Información propia complementada con CONACYT, 1992, 1993.

Si se toma otro indicador para medir las repercusiones de la investigación de la UACh, sería las líneas de investigación construidas en los distintos DEIS, sobre todo su desarrollo por lo menos cuantitativamente. 


\section{CUADRO 8}

\section{LÍNEAS DE INVESTIGACIÓN POR DEIS. 19901994.}

\begin{tabular}{lcc}
\hline & 1990 & 1994 \\
\hline CENTROS REGIONALES & 5 & 5 \\
\hline DICIFO. & N.P & \\
\hline ECONOMÍA AGRÍCOLA & 7 & 7 \\
\hline FITOTECNIA & N.P. & \\
\hline INDUSTRIAS AGRÍCOLAS & 10 & 13 \\
\hline PREPARATORIA AGRÍCOLA & 15 & 24 \\
\hline IRRIGACIÓN & N.P. & \\
\hline MAQUINARIA AGRÍCOLA & 5 & 9 \\
\hline P ARASITOLOGÍA & 11 & \\
\hline SOCIOLOGÍA RURAL & N.P. & \\
\hline ZOOTECNIA 153 & & \\
\hline
\end{tabular}

FUENTE: Resumen hecho a partir del trabajo de: Pérez Villalba, Elba, "La problemática de la investigación en la UACh", ponencia enviada al VII Foro Nacional sobre Docencia, Investigación y Servicio en el Medio Rural, Septiembre 1994, Universidad de Morelos.

Exclusivamente para el postgrado de la UACh también es relevante mencionar la cantidad de líneas desarrolladas (véase el Cuadro 9). 


\section{CUADRO 9}

\section{TOTAL DE LÍNEAS DE INVESTIGACIÓN DEL POSTGRADO. UACh.}

\begin{tabular}{lc}
\hline PROGRAMA 1993 & NUMERO \\
\hline CIENCIAS FORESTALES & 12 \\
\hline DESARROLLO RURAL & 4 \\
\hline DOC. EN ECONOMÍA AGRÍCOLA & 4 \\
\hline ECONOMÍA DESARROLLO RURAL & 4 \\
\hline HORTICULTURA & 10 \\
\hline PRODUCCIÓN ANIMAL & 8 \\
\hline PROTECCIÓN VEGETAL & 16 \\
\hline SOCIOLOGÍA RURAL & 4 \\
\hline
\end{tabular}

FUENTE: COSI UACh, 19901992.

\section{TENDENCIAS DE LA INVESTIGACIÓN DE LA UACh}

Me parece que una de las tendencias más probables de la investigación es reforzar la dirección hacia el ámbito nacional. Es decir, que la UACh de mantener su continuismo de descentralización de la investigación no sólo en el aspecto geográfico propiamente dicho sino, fundamentalmente en el terreno operativo que implica también la desconcentración en la toma de decisiones para las políticas de investigación.

Por la característica de una universidad nacional descentralizada y su organización académica vía los Centros Regionales como instancias de apoyo a la docencia e investigación, dan a la UACh una tendencia más nacional, pero sobre todo autónoma. La autonomía universitaria en la investigación implica el respeto a los programas, líneas y proyectos que son desarrollados por los investigadores de la UACh. Respecto al asunto del financiamiento para la investigación, en la medida que no se hipotequen ideológicamente los proyectos, estos pueden ser autofinanciados, cofinanciados o financiados por organismos externos a la UACh. La tendencia de la investigación de la UACh hacia el estudio de problemas nacionales en sus respectivas regiones y entidades como ya se observa en el Cuadro 10, desde 1990, es una tendencia a incrementarse a pesar de los pocos apoyos que se le otorga. 


\section{LA INVESTIGACIÓN DE VANGUARDIA TECNOLÓGICA}

De lo que sí se conoce es que la investigación en la UACh, sobre todo el presupuesto por proyectos de investigación, se ha concentrado en algunos años en ciertos DEIS. Por ejemplo entre 1980 y 1990, cinco DEIS concentran el 50\% de la investigación: Fitotecnia, Parasitología, Centros Regionales, Preparatoria y Zootecnia. En 1992, algunas de esas mismas instancias concentran más del $50 \%$ de la investigación: Centros Regionales, Fitotecnia, Economía, Irrigación y Parasitología. En la mayoría de estas investigaciones hay muy pocos trabajos que se ubiquen en la llamada Vanguardia Tecnológica.

Me parece que el cambio tecnológico (Dridrikson, 1988) o la llamada tercera revolución científico técnica (Zepeda, 1990) y lo que denominamos como uno de los retos más severos del siglo XXI (Victorino, 1992), a todas las universidades de América Latina nos agarró en una gran desventaja. Se puede decir que no existe un currículum a nivel licenciatura que esté pensado para la incorporación rápida de los avances científico tecnológicos, pues como se sabe este avance es muy rápido y al exigir actualización a los procesos de trabajo también afecta a los currículos universitarios.

CUADRO 10

DISTRIBUCIÓN GEOGRÁFICA POR ENTIDAD FEDERATIVA DE LA INVESTIGACIÓN EN LA UACh EN EL AÑO DE 1990.

\begin{tabular}{lcc}
\hline ESTADO & $\begin{array}{c}\text { NUMERO DE } \\
\text { PROYECTOS }\end{array}$ & $\%$ \\
\hline AUN SIN CLAVE & 4 & \\
\hline CON CLAVE & 449 & 100.0 \\
\hline 01. AGUASCALIENTES & 2 & 0.45 \\
\hline 02. BAJA CALIFORNIA NORTE & 2 & 0.45 \\
\hline 03. BAJA CALIFORNIA SUR & 2 & 0.45 \\
\hline 04. CAMPECHE & 4 & 0.89 \\
\hline 05. COAHUILA & 5 & 1.11 \\
\hline 06. COLIMA & 2 & 0.45 \\
\hline
\end{tabular}


Convergencia Revista de Ciencias Sociales, núm. 8/9, 1995, Universidad Autónoma del Estado de México

\begin{tabular}{lcc}
\hline 07. CHIAPAS & 10 & 2.23 \\
\hline 08. CHIHUAHUA & 5 & 1.11 \\
\hline 09. DISTRITO FEDERAL & & \\
\hline 10. DURANGO & 44 & 9.80 \\
\hline 11. GUANAJUATO & 14 & 3.12 \\
\hline 12. GUERRERO & 4 & 0.89 \\
\hline 13. HIDALGO & 9 & 2.00 \\
\hline 14. JALISCO & 9 & 2.00 \\
\hline 15. ESTADO DE MÉXICO & 192 & 42.76 \\
\hline 16. MICHOACÁN & 13 & 2.90 \\
\hline 17. MORELOS & 9 & 2.00 \\
\hline 18.NAYARIT & 2 & 0.45 \\
\hline 19. NUEVO LEÓN & 3 & 0.67 \\
\hline 20. OAXACA & 14 & 3.12 \\
\hline 21. PUEBLA & 10 & 2.23 \\
\hline 22. QUERÉTARO & 2 & 0.45 \\
\hline 23. QUINTANA ROO & 3 & 0.67 \\
\hline 24. SAN LUIS POTOSÍ & 4 & 0.89 \\
\hline 25. SINALOA & 1 & 0.22 \\
\hline 26. SONORA & 6 & 1.34 \\
\hline 27. TABASCO & 18 & 4.01 \\
\hline 28. TAMAULIPAS & 1 & 0.22 \\
\hline 29. TLAXCALA & 27 & 0.45 \\
\hline 30. VERACRUZ & 5 & 6.01 \\
\hline 31. YUCATÁN & 0 & 1.11 \\
\hline 32. ZACATECAS & 533 \\
\hline 33. DE CARÁCTER NACIONAL & 5.11 \\
\hline 34. INTERNACIONALES & & \\
\hline TOTALES: & 500.00 \\
\hline
\end{tabular}

FUENTE: UPOM, UACh, 1990. 
De tal manera que pensar en impulsar una investigación de vanguardia tecnológica en las condiciones actuales y sobre todo ante fenómenos como la internacionalización de la ciencia y la competitividad mundial es una quimera. Es necesario para la UACh repensar el paradigma de vanguardia tecnológica o lo que llamaría "innovación tecnológica" a partir de lo nacional, con el objeto de que más que interesamos por seguir incrementando la multidependencia tecnológica, habría que priorizar la ciencia y la tecnología apropiada y de interés para resolver los grandes problemas nacionales de las mayorías pobres.

Esto de ninguna manera soslaya y desconoce que existen proyectos en la UACh que estén investigando sobre ingeniería genética, o biotecnología o cualquier otro proyecto ubicado en las nuevas áreas tecnológicas, que al parecer se presentan como líneas hegemónicas que no deben desconocerse.

\section{CONCLUSIONES}

Una de las primeras conclusiones temporales del estudio nos lleva a reconocer que en materia de investigación cada institución cuenta con sus propias áreas de conocimiento o para la generación de conocimientos. Algunas de estas coinciden con cierta propuesta del CONACYT, pero en otras definitivamente responde a la tradición institucional y poco abierta a las nociones y formas nuevas que trae consigo el concepto de Universidad.

Definitivamente existe la tendencia que afianza la fuerte dependencia científico tecnológica de los países desarrollados, además existe también una fuerte influencia del positivismo como método universal de investigación sin importar la existencia y proliferación de otros enfoques o paradigmas en el tratamiento de los procesos de investigación, diferenciando para ello la distinción de ciencias, disciplinas y áreas de cono- cimientos, sobre todo de las ciencias básicas y las ciencias sociales.

De inmediato resalta una gran duda, respecto a las tendencias ante nuevos fenómenos como es la globalización económica mundial y su consecuente bloquización de regiones. Ante esto la crisis de la investigación en la UACh tiene que reconocer este nuevo reto y buscar las mejores opciones para tener un alcance de Universidad Nacional para el medio rural. 